\title{
Cloning and identification of the ASIP gene in Chinese raccoon dog (Nyctereutes procyonoides procyonoides)
}

\author{
Y.M. Li, S. Si, P.C. Guo, L.L. Li, C.Y. Bai and S.Q. Yan \\ College of Animal Science, Jilin University, Changchun, China \\ Corresponding author: S.Q. Yan \\ E-mail: yansq@jlu.edu.cn
}

Genet. Mol. Res. 14 (4): 16312-16316 (2015)

Received August 2, 2015

Accepted October 11, 2015

Published December 8, 2015

DOI http://dx.doi.org/10.4238/2015.December.8.22

\begin{abstract}
The quantity, quality, and distribution of eumelanin and pheomelanin determine a wide variety of coat colors in animals. Three coat color variants exist in farmed wild-type Chinese raccoon dog (Nyctereutes procyonoides procyonoides), which is an important fur-bearing animal species. The ASIP gene is an important candidate gene for coat color variation in some species. In this study, the complete cDNA sequences of $A S I P$ were amplified from a wild-type Chinese raccoon dog. Sequence analysis revealed the coding region of ASIP in Chinese raccoon dog to be 396-bp in length and two transcripts (accession Nos. KT224450 and KT224451) were identified due to the alternative use of exon 1 (1A and 1C). However, the alternative splicing pattern and the coding sequence of ASIP in three types of coat color variants were the same as those identified in the wild-type individual. Based on the results obtained in this study, we can exclude a role for alternative splicing of exon 1 and the coding sequence of $A S I P$ in coat color variation in Chinese raccoon dog.
\end{abstract}

Key words: Chinese raccoon dog; Coat color; ASIP gene; Alternative splicing 


\section{INTRODUCTION}

Diversity of mammalian coat color arises through the differential expression and regional distribution of eumelanin (brown/black) and pheomelanin (red/yellow). The switch between eumelanin and pheomelanin synthesis is mainly controlled by the products encoded by the MC1R and ASIP genes. The ASIP gene encodes the agouti signaling protein, which is involved in the switch from eumelanin to pheomelanin synthesis in melanocytes. Mutations or polymorphisms in the ASIP gene have been implicated in coat color phenotype in a variety of animals including mice (Vrieling et al., 1994; Hustad et al., 1995), pigs (Drögemüller et al., 2006), sheep (Norris and Whan, 2008; Li et al., 2014), goats (Badaoui et al., 2011), horses (Rieder et al., 2001), rabbits (Fontanesi et al., 2010), red foxes (Våge et al., 1997), and dogs (Dreger and Schmutz, 2011; Ciampolini et al., 2013).

The Chinese raccoon dog (Nyctereutes procyonoides procyonoides), which has a mixed coat color of black-to-brown with grey hairs (Han et al., 2012), is an important fur-bearing animal species worldwide (Nowacka-Woszuk et al., 2013; Yan et al., 2013). Nowadays, three coat color variants exist in farmed wild-type Chinese raccoon dog (Figure 1), which are brown, dominantwhite, and black. Because the mutation responsible for these variants in Chinese raccoon dog remains unknown, the aim of this study was to clone and analyze cDNA sequences of the ASIP gene from animals presenting four coat-color phenotypes, and to further determine whether these phenotypes are associated with polymorphisms in this species.

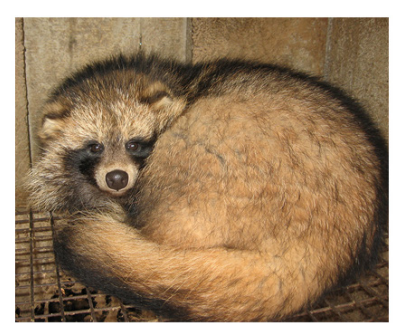

A

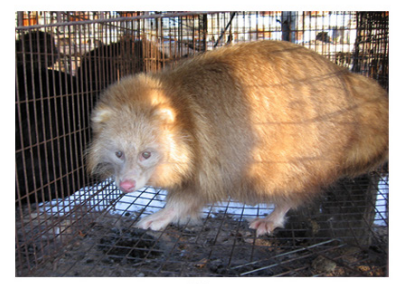

C

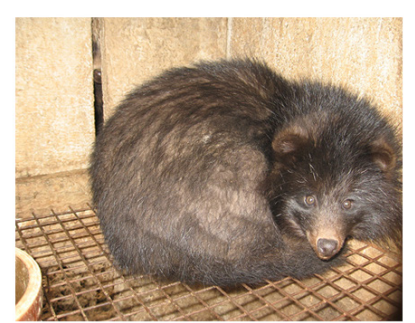

B

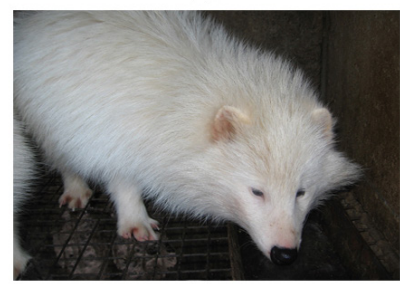

D

Figure 1. Coat-color phenotypes of the adult Chinese raccoon dogs in winter. A. wild-type; B. black; C. brown; D. dominant-white.

\section{MATERIAL AND METHODS}

Skin tissues were taken from four farmed Chinese raccoon dogs presenting four types of coat color from three fur-bearing farms in Shandong, Hebei, and Jilin, China. Animal experiments were performed in accordance with the guidelines for animal care established by the Jilin University Animal Care and Use Committee. Total RNA was extracted using the RNAprep pure Tissue Kit (TIANGEN, Beijing, China) according to the manufacturer protocol and immediately frozen at 
$-80^{\circ} \mathrm{C}$. The RNA concentration was quantified using an ultraviolet spectrophotometer, and RNA quality was assessed on $1.0 \%$ agarose gels.

In order to obtain the full-length cDNA sequence of ASIP from Chinese raccoon dog, 5 ' and 3' rapid-amplification of cDNA ends (RACE) was carried out according to the protocol reported by Cosentino et al. (2010). Gene-specific primers were designed based on the canine ASIP mRNA sequence (GenBank accession No. NM_001007263). In 5' RACE, first-strand cDNA was synthesized from total RNA using a PrimeScript ${ }^{T M}$ RT reagent Kit (TaKaRa, Dalian, China) following the manufacturer instructions. A poly $(\mathrm{dC})$ tail was then added to the 3 ' end of the cDNA using TdT terminal transferase (TaKaRa). The first round of PCR was performed with 5-AP (5'-GGCCACGCGTCGACTAGTACGGGGGGGGGGGGGGG-3') and HAP-R1 (5'- TTCTTTTCCG CCTCTTTTCTGCT-3') as primers. PCR was performed in a $25-\mu \mathrm{L}$ reaction under the following conditions: $95^{\circ} \mathrm{C}$ for $2 \mathrm{~min} ; 30$ cycles of $94^{\circ} \mathrm{C}$ for $30 \mathrm{~s}, 62^{\circ} \mathrm{C}$ for $30 \mathrm{~s}$, and $72^{\circ} \mathrm{C}$ for $1 \mathrm{~min}$; and a final extension at $72^{\circ} \mathrm{C}$ for $5 \mathrm{~min}$. Nested PCR was carried out using 5-AP1 (5'-GGCCACGCGTCGACTAGTAC-3') and HAP-R2 (5'-AGACAGAAGGGAAATCCAAAAGG-3') as primers and $0.5 \mu \mathrm{L}$ of the product from the first-round of PCR as the template. The PCR components and reaction conditions were the same as those described above. In 3' RACE, first-strand cDNA was synthesized using 3-AP1 (5'-GGCCACGCGTCGACTAGTACTTTTTTTTTTTTTTTTT-3') as a primer. PCR was carried out with HAP-F1 (5'-TTCCTCACTGCCTACAGCCAC-3') and 3-AP1 (5'-GGCCACGCGTCGACTAGTAC-3') as primers under the same conditions described for $5^{\prime}$ RACE. PCR products were separated on $1.5 \%$ agarose gels, and the fragments were extracted with a gel extraction kit (Axygen, Hangzhou, China) and cloned into a pMD-18T vector according to the manufacturer protocol. Clones containing inserts were sequenced by Sangon Biological Company (Shanghai, China).

In order to investigate polymorphisms in the coding region of ASIP, and in the two versions of exon 1 in Chinese raccoon dogs with different coat color phenotypes, two forward primers (HAP1A-F: 5'-TTCACAGACCCTTCCAACTCAG-3'; HAP-1C-F: 5'-GACAGGGAAGACGCTGAGCT-3') and a reverse primer (HAP-R3: 5'-GGGATCCCCAAAGCCGTAC-3') were designed according to the cDNA sequence obtained above. RT-PCR was carried out using the primer sets HAP-1A-F/ HAP-R3 and HAP-1C-F/HAP-R3, respectively. PCR products were purified and directly sequenced.

\section{RESULTS AND DISCUSSION}

Using RACE methods, the 5' and $3^{\prime}$ ends of ASIP from Chinese raccoon dog were amplified successfully from the skin tissue of a wild-type individual (Figure 2). After gel extraction and purification, the products were ligated into a pMD-18T vector, and positive clones were subsequently sequenced. One 583-bp fragment (excluding the polyA tail) was obtained by 3' RACE. However, two fragments (243 and 235 bp) were obtained by 5' RACE amplification (Figure 3). BLASTN analysis using the NCBI GenBank database (http://www.ncbi.nlm.nih.gov) indicated the existence of two versions of exon 1 , designated $1 \mathrm{~A}$ and $1 \mathrm{C}$ according to their high identity with the $1 \mathrm{~A}$ and $1 \mathrm{C}$ counterparts in dog (Ciampolini et al., 2013). By aligning the $1 \mathrm{~A}$ and $1 C$ sequences of $A S I P$ from Chinese raccoon dog with a canine genomic DNA sequence in GenBank (NC_006606), they were found to be located at positions 23338843-23338927 and 23378757-23378833, respectively. Therefore, $1 \mathrm{~A}$ and $1 \mathrm{C}$ are generated by transcription initiated at different sites. 


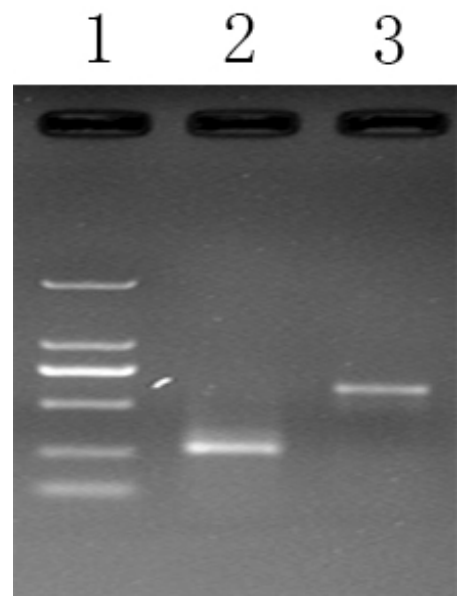

Figure 2. Agarose gel electrophoresis of PCR products. Lane 1, DL2000 marker. Lane 2, 5'-RACE amplification using primer set 5-AP1 and HAP-R2. Lane 3, 3'-RACE amplification using primer set HAP-F1 and 3-AP1.

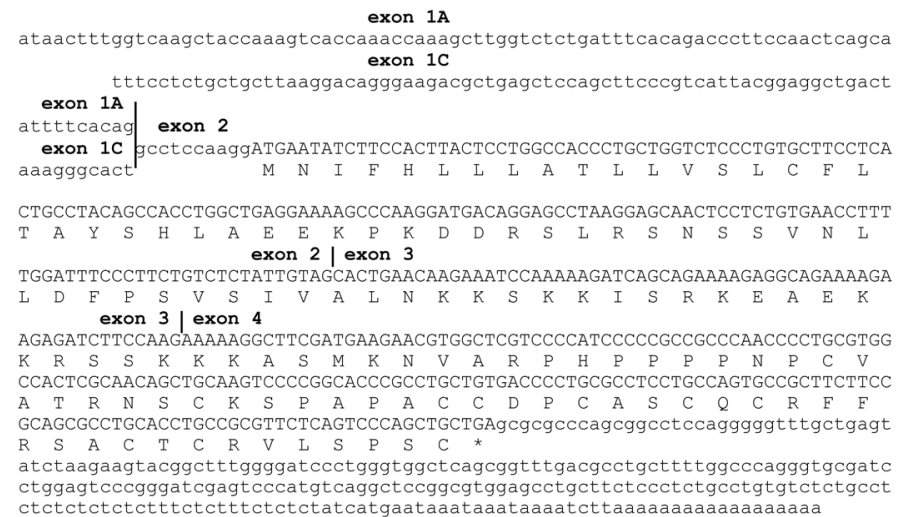

Figure 3. cDNA and deduced amino acid sequences of ASIP from Chinese raccoon dogs. The vertical lines mark the junctions of exons with the exon numbers. The stop codon is represented by the asterisk. Underlined text indicates the probable polyadenylation signal.

By assembling the cloned $3^{\prime}-$ and 5 '-end sequences obtained by RACE, two full-length cDNAs of ASIP from Chinese raccoon dog were generated. The transcripts of ASIP are 726- and 718-bp long excluding the poly (A) tail, and contain a 396-bp open reading frame, a 95-bp (87-bp for 1C) 5'-untranslated region (UTR), and a 235-bp 3'-UTR. These sequences have been deposited in NCBI GenBank under accession No. KT224450 and KT224451, respectively. The ASIP of Chinese raccoon dog encodes a putative protein containing 131 amino acids, which is 99.2, 97.7, 82.2, 81.4, and 81.4\% identical to that of red fox (accession No. P79407), dog (NP_001007264), cattle (NP_996674), mouse (NP_056585), and human (NP_001663), respectively. According to the genomic sequence of canine ASIP (NC_006606) and mRNA data obtained in this study, we can deduce that the ASIP gene in Chinese raccoon dog is composed of four exons and three introns. 
RT-PCR amplification using primer sets HAP-1A-F/HAP-R3 and HAP-1C-F/HAP-R3 produced 502- and 527-bp fragments from three other individuals representing three types of coatcolor variations. Sequencing revealed that two transcripts resulting from the alternative splicing of exon 1 ( $1 \mathrm{~A}$ and $1 \mathrm{C}$ ) of the ASIP gene were present in three types of coat-color variants. Therefore, we excluded a possible association between alternative splicing and coat-color variation in Chinese raccoon dog. In addition, no polymorphism was found in the coding region of the ASIP gene among any individuals examined in this study.

\section{Conflicts of interest}

The authors declare no conflict of interest.

\section{ACKNOWLEDGMENTS}

Research supported by a project from the National Natural Science Foundation of China (\#31401979) and the Fundamental Research Funds of Jilin University (\#200903332).

\section{REFERENCES}

Badaoui B, D'Andrea M, Pilla F, Capote J, et al. (2011). Polymorphism of the goat agouti signaling protein gene and its relationship with coat color in Italian and Spanish breeds. Biochem. Genet. 49: 523-532.

Ciampolini R, Cecchi F, Spaterna A, Bramante A, et al. (2013). Characterization of different 5'-untranslated exons of the ASIP gene in black-and-tan Doberman Pinscher and brindle Boxer dogs. Anim. Genet. 44: 114-117.

Cosentino C, Fischer-Schliebs E, Bertl A, Thiel G, et al. (2010). Na+/H+ antiporters are differentially regulated in response to $\mathrm{NaCl}$ stress in leaves and roots of Mesembryanthemum crystallinum. New Phytol. 186: 669-680.

Dreger DL and Schmutz SM. (2011). A SINE insertion causes the black-and-tan and saddle tan phenotypes in domestic dogs. J. Hered. 102: S11-S18.

Drögemüller C, Giese A, Martins-Wess F, Wiedemann S, et al. (2006). The mutation causing the black-and-tan pigmentation phenotype of Mangalitza pigs maps to the porcine ASIP locus but does not affect its coding sequence. Mamm. Genome 17: 58-66.

Fontanesi L, Forestier L, Allain D, Scotti E, et al. (2010). Characterization of the rabbit agouti signaling protein (ASIP) gene: transcripts and phylogenetic analyses and identification of the causative mutation of the nonagouti black coat color. Genomics 95: 166-175.

Han JI, Yang H, Jeung EB and Na KJ (2012). Altered expression of melanocortin-1 receptor (MC1R) in a yellow-coloured wild raccoon dog (Nyctereutes procyonoides). Vet. Dermatol. 23: 187-e37.

Hustad CM, Perry WL, Siracusa LD, Rasberry C, et al. (1995). Molecular genetic characterization of six recessive viable alleles of the mouse agouti locus. Genetics 140: 255-265.

Li MH, Tiirikka T and Kantanen J (2014). A genome-wide scan study identifies a single nucleotide substitution in ASIP associated with white versus non-white coat-color variation in sheep (Ovis aries). Heredity 112: 122-131.

Norris BJ and Whan VA. (2008). A gene duplication affecting expression of the ovine ASIP gene is responsible for white and black sheep. Genome Res. 18: 1282-1293.

Nowacka-Woszuk J, Salamon S, Gorna A and Switonski M (2013). Missense polymorphisms in the MC1R gene of the dog, red fox, arctic fox and Chinese raccoon dog. J. Anim. Breed. Genet. 130: 136-141.

Rieder S, Taourit S, Mariat D, Langlois B, et al. (2001). Mutations in the agouti (ASIP), the extension $(M C 1 R)$, and the brown (TYRP1) loci and their association to coat color phenotypes in horses (Equus caballus). Mamm. Genome 12: 450-455.

Våge DI, Lu D, Klungland H, Lien S, et al. (1997). A non-epistatic interaction of agouti and extension in the fox, Vulpes vulpes. Nat. Genet. 15: 311-315.

Vrieling H, Duhl DM, Millar SE, Miller KA, et al. (1994). Differences in dorsal and ventral pigmentation result from regional expression of the mouse agouti gene. Proc. Natl. Acad. Sci. USA 91: 5667-5671.

Yan SQ, Li YM, Bai CY, Ding XM, et al. (2013). Development and characterization of polymorphic microsatellite markers for Chinese raccoon dog (Nyctereutes procyonoides procyonoides). Genet. Mol. Res. 12: 6351-6355. 